\title{
A connection problem involving a logarithmic function
}

\author{
By \\ Kenjiro Окuво \\ (Faculty of Science and Technology, Sophia University, Tokyo)
}

A two points connection problem between two sets of fundamental solutions for a system of ordinary differential equations $t d X /$ $d t=(A+t B) X$ is studied under the assumptions that the eigenvalues $\lambda_{k}(k=1,2, \cdots, n)$ of the diagonal matrix $B$ satisfy $\left|\lambda_{j}-\lambda_{k}\right|>\left|\lambda_{k}\right|>0$, and that the matrix $A$ has a pair of congruent eigenvalues. Connection coefficients are calculated by convergent series and error terms are reduced to be asymptotically zero.

1. Introduction. In a preceding paper we have studied the relation between two fundamental sets of solutions of a system of $n$ linear ordinary differential equations of the form,

$$
t \frac{d X}{d t}=(A+t B) X
$$

under the conditions that no two eigenvalues of the $n$ by $n$ constant matrix $A$ are congruent modulo 1 , and that the $n$ by $n$ constant diagonal matrix $B$ has no pair of eigenvalues with coincident arguments.

One set of solutions has convergent power series expansion at the origin. expressed by

$$
X_{j}(t)=t^{\rho}{ }_{j} \sum_{m=0}^{\infty} G_{j}(m) t^{m} \quad(j=1,2, \cdots, n)
$$

where $\rho_{j}(j=1,2, \cdots, n)$ are eigenvalues of the matrix $A$. The other

Received January 19, 1965

Communicated by M. Hukuhara

*) The research was partly sponsored by The Sakkōkai Foundation. 
set consists of those solutions which are expressed asymptotically, in a certain sectorial neighborhood of the infinity, by the following formal solutions :

$$
X^{k}(t) \sim x^{k}(t) \sum_{s=0}^{\infty} H^{k}(s) t^{-s}
$$

with the scalar function $x^{k}(t)(k=1,2, \cdots, n)$ defined by

$$
x^{k}(t)=\exp \left(\lambda_{k} t\right) t^{a}{ }_{k k}
$$

where $a_{k k}$ is the $k$-th diagonal element of $A$ and $\lambda_{k}$ is that of $B$.

The chief object of the present investigation is to extend our study to the case where $A$ has a pair of congruent eigenvalues, and accordingly convergent expressions involve a logarithmic function. For the sake of definiteness, we write this pair $\left(\rho_{1}, \rho_{2}\right)$, and we assume that $\rho_{2}-\rho_{1}=m_{0}$ is a positive integer. We, also, assume that none of the quantities $a_{k k}-\rho_{j}(j, k=1,2, \cdots, n)$ is a nonnegative integer.

To make the discussions simpler, and at the same time to prepare for the more complicated situations in the cases of irregular singularities of higher rank, we make the following assumption on the eigenvalues $\lambda_{1}, \lambda_{2}, \cdots, \lambda_{n}$ of $B$ :

$$
\left|\lambda_{j}-\lambda_{k}\right|>\left|\lambda_{j}\right|>0 \quad(j \neq k)
$$

We shall briefly refer this condition as the pentagonal condition, because a polygon formed in complex $\lambda$-plane with vertices $\lambda_{1}, \lambda_{2}$, $\cdots, \lambda_{n}$ is at most pentagonal for (1.5) to be satisfied. This is easily seen if one draws an ortho-hexagon with center at the origin and labels the six vertices $\lambda_{1}, \cdots, \lambda_{6}$. The significance of the pentagonal condition lies in the fact that no procedure of analytic continuation is necessary in the calculation of connection coefficients. (See the final section.)

Naturally, our theory is in a very close connection with the canonicalization of more general systems of the form

$$
t \frac{d Y}{d t}=\left(t^{q} \sum_{r=0}^{\infty} A_{r} t^{-r}\right) Y
$$

We refer the reader to the survey article by H.L. Turrittin, [5]. 
Essentially, the method employed here is the one in our preceding paper [2]. But the treatment is aimed to be applicable to the case of higher rank singularity, consequently we used no results from the preceding paper. Moreover we made a small progress in eliminating the error term $O\left(t^{h}\right)$ which appeared in our connection formula in the preceding paper.

We usually denote vector valued variables by capital Italics, and scalars by small Italics. " $I$ " to denote the identity matrix is suppressed when it is multiplied by a scalar, e.g., $(\lambda-B)$ should be $\operatorname{read}(\lambda I-B)$.

\section{Single inhomogeneous equations}

Proposition 2.1. If $r(t)$ is holomorphic and $t^{l} r(t)$ is bounded for a real number $l$ such that $\Re(\alpha+l)>0$, in

$$
\begin{aligned}
\mathfrak{D}^{*} & =\left\{t ;|t| \geqq t_{0}>0,|\operatorname{argt} t| \leqq \frac{3}{2} \pi-\eta\right\} \\
& -\left\{t ;|\arg t|>\pi, \Re t<-\left|t_{0}\right|\right\}
\end{aligned}
$$

where $\eta$ is an arbitrarily small positive real number, then a solution $y(t)$ of

$$
t \frac{d y}{d t}=(t+\alpha) y+r(t)
$$

bounded in (2.1), has the form

$$
y(t)=0\left(t^{-l}\right)
$$

in $\mathfrak{D} *$.

Proof. We prove the proposition only for $t$ with non-negative argument, the remaining case will be proved similarly.

Let us consider a path $P_{t^{\prime}}$, depending on a positive parameter $t^{\prime}$, which consists of the following three parts :
(i ) straight line$$
\tau=-t^{\prime}+i \mu
$$$$
(-\infty<\mu<0)
$$
(ii) semi-circle
$\tau=t^{\prime} \exp (i(\pi-\mu))$
$(0 \leqq \mu<\pi)$
(iii) positive real axis $\tau=\frac{t^{\prime}}{\pi} \mu$
$(\pi \leqq \mu)$. 
The positive orientation of the path is taken in the increasing sense of $\mu$. Obviously, for any $t$ in $\mathfrak{D}^{*}$ with non-negative argument, there is exactly one $t^{\prime}$ for which $t$ lies on $P_{t^{\prime}}$, if we define:

$$
\begin{aligned}
t^{\prime} & =-\Re t & & (\pi<\arg t) \\
& =|t| & & (0 \leq \arg t \leq \pi)
\end{aligned}
$$

Consider the general solution of the equation (2.1) obtained by quadrature.

$$
y(t)=c e^{t} t^{\alpha}-\int_{t}^{\infty} e^{t-\tau} r(\tau)\left(\frac{t}{\tau}\right)^{\alpha} \frac{d \tau}{\tau}
$$

We will first prove the boundedness of

$$
-t^{l} \int_{t}^{\infty} e^{t-\tau} r(\tau)\left(\frac{t}{\tau}\right)^{\alpha} \frac{d \tau}{\tau}=-\int_{t}^{\infty} e^{t-\tau}\left(\frac{t}{\tau}\right)^{a+l} \tau^{l} r(\tau) \frac{d \tau}{\tau}
$$

in $\mathfrak{D}^{*}$ when the integration is carried out along the path $P_{t^{\prime}}$. Then we must necessarily have $c=0$ in the general solution in order that $y(t)$ is bounded in $\mathfrak{D}^{*}$. Thus we have

$$
t^{l} y(t)=-\int_{t}^{\infty} e^{t-\tau}\left(\frac{t}{\tau}\right)^{a+l} \tau^{l} r(\tau) \frac{d \tau}{\tau}
$$

and the proof will follow from the boundedness of the integral which we are going to show.

We observe from the figure the following estimates shown in the table below for the three quantities in the first column on the path of integration.

\begin{tabular}{|c|c|c|c|c|c|c|}
\hline & \multicolumn{3}{|c|}{$\arg t>\pi$} & \multicolumn{2}{|c|}{$0<\arg t \leqq \pi$} & $\arg t=0$ \\
\hline & $\begin{array}{c}\text { (i) } \\
\operatorname{Im} t \leqq \mu<0\end{array}$ & $\begin{array}{c}\text { (ii) } \\
0 \leqq \mu<\pi\end{array}$ & $\begin{array}{c}\text { (iii) } \\
\pi \leqq \mu>\infty\end{array}$ & $\begin{array}{c}\text { (ii) } \\
\pi-\arg t \leqq \mu<\pi\end{array}$ & $\begin{array}{c}\text { (iii) } \\
\pi \leqq \mu<\infty\end{array}$ & $\begin{array}{c}\text { (iii) } \\
\pi \leqq \mu<\infty\end{array}$ \\
\hline$\left|e^{t-\tau}\right|$ & $=1$ & $\leqq 1$ & $\leqq e^{-t^{\prime}(1+\mu / \pi)}$ & $\leqq 1$ & $\leqq e^{t^{\prime}(1-\mu / \pi)}$ & $\leqq e^{t^{\prime}(1-\mu / \pi)}$ \\
\hline$\left|\frac{t}{\tau}\right|$ & $\leqq \frac{1}{\sin \eta}$ & $\leqq \frac{1}{\sin \eta}$ & $\leqq \frac{\pi}{\sin \eta} \frac{1}{\mu}$ & $=1$ & $=\frac{\pi}{\mu}$ & $=\frac{\pi}{\mu}$ \\
\hline$\left|\frac{d \tau}{\tau}\right|$ & $\leqq \frac{d \mu}{t^{\prime}}$ & $=d \mu$ & $=\frac{d \mu}{\mu}$ & $=d \mu$ & $=\frac{d \mu}{\mu}$ & $=\frac{d \mu}{\mu}$ \\
\hline
\end{tabular}


From the above table we observe that $\left|\left(\frac{t}{\tau}\right)^{a+l}\right|$ can always be majorized by a constant and that $\left|e^{t-\tau}\right|$ can always be majosized by 1 , when $\mu<\pi$. Therefore the boundedness of the integral on the part (i) and (ii) is evident, and the whole integral will be bounded if it is bounded on the part (iii) of $P_{t^{\prime}}$. Now we have for $\arg t>\pi$.

$$
\left|\int_{(\mathrm{iii})}\right| \leqq K \int_{\pi}^{\infty} e^{-t^{\prime(1+\mu / \pi)}} \frac{d \mu}{\mu} \leqq K^{\prime} \int_{2}^{\infty} e^{-t+\mu} \frac{d \mu}{1+\mu} \leqq K^{\prime} \int_{0}^{\infty} e^{-t^{\prime} \mu} d \mu<\frac{K^{\prime}}{t_{0}}
$$

and for $0 \leqq \arg t \leq \pi$.

$$
\left|\int_{(\mathrm{iii})}\right| \leqq K \int_{\pi}^{\infty} e^{t^{\prime}(1-\mu / \pi)} \frac{d \mu}{\mu} \leqq K \int_{0}^{\infty} e^{-t^{\prime} \mu} \frac{d \mu}{1+\mu} \leqq \frac{K}{t_{0}}
$$

This completes the proof.

Definition 2.1. $x_{j}(t)(j=1,2)$ are holomorphic solutions at the origin of

$$
\left\{\begin{array}{l}
t \frac{d x_{1}}{d t}=(t+\alpha) x_{1}+\frac{1}{\Gamma(-\alpha)} \\
t \frac{d x_{2}}{d t}=(t+\alpha) x_{2}-x_{1}
\end{array}\right.
$$

where $\alpha$ is not a non-negative integer. We shall write these solutions as

$$
x_{j}(t)=\sum_{m=0}^{\infty} g_{j}(m) t^{m} \quad(j=1,2)
$$

Proposition 2.2. We have

$$
g_{1}(m)=\frac{1}{\Gamma(m-\alpha+1)}
$$

and

$$
g_{2}(m)=\frac{\psi(-\alpha)-\psi(m-\alpha+1)}{\Gamma(m-\alpha+1)}
$$

where $\psi(z)$ is the di-gamma function defined by

$$
\psi(z)=\frac{d}{d z}\{\log \Gamma(z)\}
$$


Proof. $\psi(z)$ satisfies the difference equation

$$
\psi(z+1)-\psi(z)=\frac{1}{z}
$$

With the help of (2.9) one can verify the proposition easily by direct substitution of (2.5) into (2.4).

Proposition 2. 3. Given any positive integer $\sigma$, and $t$ in $\mathfrak{D}^{*}$, we have

$$
x_{1}(t)=e^{t} t^{\alpha}-\sum_{l=1}^{\sigma} g_{1}(-l) t^{-l}+0\left(t^{-\sigma}\right)
$$

and

$$
x_{2}(t)=\psi(-\alpha) e^{t} t^{\alpha}-\log t e^{t} t^{\alpha}-\sum_{l=1}^{\sigma} g_{2}(-l) t^{-l}+0\left(t^{-\sigma}\right) .
$$

Proof. First suppose that $\Re \alpha<0$. Solving (2.4) by quadrature. we immediately see that, in order that $x_{j}(t)$ be holomorphic at the origin. they must be of the form

$$
\begin{aligned}
x_{1}(t) & =\frac{1}{\Gamma(-\alpha)} \int_{0}^{t} e^{t-\tau}\left(\frac{t}{\tau}\right)^{\alpha} \frac{d \tau}{\tau}=\frac{1}{\Gamma(-\alpha)} \int_{0}^{\infty} e^{t-\tau}\left(\frac{t}{\tau}\right)^{\alpha} \frac{d \tau}{\tau} \\
& -\frac{1}{\Gamma(-\alpha)} \int_{t}^{\infty} e^{t-\tau}\left(\frac{t}{\tau}\right)^{\alpha} \frac{d \tau}{\tau}=e^{t} t^{\alpha}-\frac{1}{\Gamma(-\alpha)} \int_{t}^{\infty} e^{t-\tau}\left(\frac{t}{\tau}\right)^{\alpha} \frac{d \tau}{\tau} \\
x_{2}(t) & =-\int_{0}^{t} e^{t-\tau}\left(\frac{t}{\tau}\right)^{\infty} x_{1}(\tau) \frac{d \tau}{\tau}=-\frac{1}{\Gamma(-\alpha)} \int_{0}^{t} \frac{d \tau}{\tau}\left[\int_{0}^{\tau} e^{t-\mu}\left(\frac{t}{\mu}\right)^{\alpha} \frac{d \mu}{\mu}\right] \\
& =-\frac{1}{\Gamma(-\alpha)} \int_{0}^{t} e^{t-\tau}\left(\frac{t}{\tau}\right)^{\alpha} \log \left(\frac{t}{\tau}\right) \frac{d \tau}{\tau} \\
& =-e^{t} t^{\alpha} \log t+\psi(-\alpha) e^{t} t^{\alpha}+\frac{1}{\Gamma(-\alpha)} \int_{t}^{\infty} e^{t-\tau}\left(\frac{t}{\tau}\right)^{\infty} \log \left(\frac{t}{\tau}\right) \frac{d \tau}{\tau}
\end{aligned}
$$

where we used the defirition (2.8) of $\psi(z)$ in the form

$$
\psi(z)=\frac{d}{d z} \log \Gamma(z)=\frac{1}{\Gamma(z)} \frac{\partial}{\partial z} \int_{0}^{\infty} e^{-\tau} \tau^{z-1} d \tau
$$

Consider the integrals on the right, when integrated by parts once.

$$
\int_{t}^{\infty} e^{t-\tau}\left(\frac{t}{\tau}\right)^{\alpha} \frac{d \tau}{\tau}=\frac{1}{t}-\frac{\alpha+1}{t} \int_{t}^{\infty} e^{t-\tau}\left(\frac{t}{\tau}\right)^{\alpha+1} \frac{d \tau}{\tau}
$$




$$
\begin{aligned}
\int_{t}^{\infty} e^{t-\tau}\left(\frac{t}{\tau}\right)^{\alpha} \log \left(\frac{t}{\tau}\right) \frac{d \tau}{\tau} & =\frac{1}{t} \int_{t}^{\infty} e^{t-\tau}\left(\frac{t}{\tau}\right)^{\alpha+1} \frac{d \tau}{\tau} \\
& -(\alpha+1) \frac{1}{t} \int_{t}^{\infty} e^{t-\tau}\left(\frac{t}{\tau}\right)^{\alpha+1} \log \left(\frac{t}{\tau}\right) \frac{d \tau}{\tau}
\end{aligned}
$$

It is clear that if we repeat the partial integrations, we will arrive at the expressions

$$
\begin{aligned}
& x_{1}(t)-e^{t} t^{\alpha} \mid=\left(\text { polynomial in } \frac{1}{t}\right) \\
& x_{2}(t)+e^{t} t^{\alpha} \log t-\psi(-\alpha) e^{t} t^{\alpha}\left\{=\left(\text { linear form in } \frac{1}{t^{\sigma}} \int_{t}^{\infty} e^{(t-\tau)}\left(\frac{t}{\tau}\right)^{\alpha+\sigma} \frac{d \tau}{\tau}\right.\right. \\
&+ \\
&\text { and } \left.\frac{1}{t^{\sigma}} \int_{t}^{\infty} e^{t-\tau}\left(\frac{t}{\tau}\right)^{\alpha+\sigma} \log \left(\frac{t}{\tau}\right) \frac{d \tau}{\tau}\right)
\end{aligned}
$$

in which all the integrals are bounded in $\mathfrak{D}^{*}$, if we take $\sigma$ sufficiently large. Motivated by these facts, we set

$$
\left\{\begin{array}{l}
x_{1}(t)=e^{t} e^{\alpha}-\sum_{l=0}^{\sigma} g_{1}(-l) t^{-l}+y_{1}(t) \\
x_{2}(t)=-e^{t} t^{\alpha} \log t+\psi(-\alpha) e^{t} t^{\alpha}-\sum_{l=1}^{\sigma} g_{2}(-l) t^{-l}+y_{2}(t)
\end{array}\right.
$$

Then $y_{j}(t)(j=1,2)$ are bounded solutions of equations

$$
\left\{\begin{array}{l}
t \frac{d y_{1}}{d t}=(t+\alpha) y_{1}-(\sigma+\alpha) g_{1}(-\sigma) t^{-\sigma} \\
t \frac{d y_{2}}{d t}=(t+\alpha) y_{2}-y_{1}(t)-g_{2}(-\sigma-1) t^{-\sigma}
\end{array}\right.
$$

Now we can apply the proposition 2.1., if $\Re \alpha+\sigma>0$, to have

$$
y_{1}(t)=0\left(t^{-\sigma}\right), \quad y_{2}(t)=0\left(t^{-\sigma}\right) \quad\left(t \in \mathfrak{D}^{*}\right)
$$

When $\Re \alpha+\sigma<0$, take a sufficiently large $\sigma^{\prime}$ such that $\Re \alpha+\sigma^{\prime}>0$ and we have

$$
\begin{aligned}
\sum_{l=1}^{\sigma} g_{j}(-l) t^{-l}+0\left(t^{-\sigma^{\prime}}\right) & =\sum_{l=1}^{\sigma} g_{j}(-l) t^{-l}+\sum_{l=\sigma_{+1}}^{\sigma^{\prime}} g_{j}(-l) t^{-l}+0\left(t^{-\sigma^{\prime}}\right) \\
& =\sum_{l=1}^{\sigma} g_{j}(-l) t^{-l}+0\left(t^{-\sigma-1}\right)
\end{aligned}
$$

This completes the proof for $\mathfrak{R} \alpha<0$.

To prove the proposition for arbitrary $\alpha$, we use the following 
uotations to clarify the dependence of $x_{j}(t)(j=1,2)$ on the parmeter $\alpha$,

$$
\begin{aligned}
x_{j}(t, \alpha) & =\sum_{m=0}^{\infty} g_{j}(m, \alpha) t^{m} \quad(j=1,2) \\
g_{1}(m, \alpha) & =\frac{1}{\Gamma(m-\alpha+1)} \\
g_{2}(m, \alpha) & =\frac{\psi(-\alpha)-\psi(m-\alpha+1)}{\Gamma(m-\alpha+1)}
\end{aligned}
$$

They satisfy the recurrence relations

$$
\left\{\begin{aligned}
x_{1}(t, \alpha+1) & =\frac{1}{\Gamma(-\alpha)}+t x_{1}(t, \alpha) \\
x_{2}(t, \alpha+1) & =\{\psi(-\alpha-1)-\psi(-\alpha)\} x_{1}(t, \alpha+1)+t x_{2}(t, \alpha)
\end{aligned}\right.
$$

We can deduce the asymptotic expansions (2.10) and (2.11) for $\alpha+1$ from those for $\alpha$ as follows :

$$
\begin{gathered}
x_{1}(t, \alpha+1)=\frac{1}{\Gamma(-\alpha)}+t e^{t} t^{\alpha}-t \sum_{l=1}^{\sigma+1} \frac{t^{-l}}{\Gamma(-l-\alpha+1)}+0\left(t^{-\sigma-1}\right) \cdot t \\
=e^{t} t^{\alpha+1}-\sum_{l=1}^{\sigma} \frac{t^{-l}}{\Gamma(-l-\alpha)}+0\left(t^{-\sigma}\right) \\
x_{2}(t, \alpha+1)=\left\{\psi(-\alpha-1)-\psi(-\alpha\}\left\{e^{t} t^{\alpha+1}-\sum_{l=1}^{\sigma} g_{1}(-l, \alpha+1) t^{-l}+0\left(t^{-\sigma}\right)\right\}\right. \\
-\log t e^{t} t^{\alpha-1}+\psi(-\alpha) e^{t} t^{\alpha+1}-t \sum_{l=1}^{\sigma+1} g_{2}(-l, \alpha) t^{-l}+0\left(t^{-\sigma}\right) \\
=-\log t \cdot e^{t} t^{\alpha-1}+\psi(-\alpha-1) e^{t} t^{\alpha+1}-\sum_{l=1}^{\sigma} g_{2}(-l, \alpha+1) t^{-l}+0\left(t^{-\sigma}\right)
\end{gathered}
$$

Our proposition is proved by repeating this process.

Definition 2.2. $x_{j}^{k}(t)(j, k=1,2, \cdots, n)$ are holomorphic solutions at $t=0$ of

$$
\left\{\begin{aligned}
t \frac{d x_{j}^{k}}{d t} & =\left(\lambda_{k}+a_{k k}-\rho_{j}\right) x_{j}^{k}+\frac{1}{\Gamma\left(\rho_{j}-a_{k k}\right)} \quad(j=2,3, \cdots, n) \\
t \frac{d x_{1}^{k}}{d t} & =\left(\lambda_{k} t+a_{k k}-\rho_{2}\right) x_{1}^{k}-x_{2}^{k}
\end{aligned}\right.
$$

where none of the numbers $a_{k k}-\rho_{j}(j, k=1,2, \cdots, n)$ is a non-negative integer, and $\rho_{2}-\rho_{1}=m_{0}$ is a positive integer.

We whall write those solutions in the form 
A Connection Problem Involving a Logarithmic Function 107

$$
x_{j}^{k}(t)=\sum_{m=0}^{\infty} g_{j}^{k}(m) t^{m}
$$

A special attention is called upon the use of $\rho_{2}$, instead of $\rho_{1}$, in the definition of $x_{1}^{k}(t)(k=1,2, \cdots, n)$.

Proposition 2. 4. The coefficients $g_{j}^{k}(m)$ are given by

$$
g_{j}^{k}(m)=\frac{\lambda_{k}^{m}}{\Gamma\left(m+\rho_{j}-a_{k k}+1\right)} \quad(j=2,3, \cdots, n)
$$

and

$$
g_{1}^{k}(m)=\frac{\psi\left(\rho_{2}-a_{k k}\right)-\psi\left(m+\rho_{2}-a_{k k}+1\right)}{\Gamma\left(m+\rho_{2}-a_{k k}+1\right)} \lambda_{k}^{m}
$$

Proof. Trivial.

Proposition 2.5. For $t$ in $\mathfrak{D}_{k}$, defined by

$$
\mathfrak{D}_{k}=\left\{t ; \lambda_{k} t \in \mathfrak{D}^{*}\right\}
$$

we have for $j=2,3, \cdots, n, k=1,2, \cdots, n$

$$
\sum_{m=0}^{\infty} g_{j}^{k}(m+s) t^{m}=\lambda_{k}{ }^{{ }^{k}{ }^{-\rho} j} x^{k}(t) t^{-\rho_{j}-s}-\sum_{l=1}^{\sigma-1} g_{j}^{k}(s-l) t^{-l}+0\left(t^{-\sigma}\right) .
$$

and for $j=1, k=1,2, \cdots, n$,

$$
\begin{aligned}
& \sum_{m=0}^{\infty} g_{l}^{k}(m+s) t^{m}=\lambda_{k}{ }^{a}{ }_{k k^{-\rho}}\left\{\psi\left(\rho_{2}-a_{k k}\right)-\log \lambda_{k} t\right\} x^{k}(t) t^{-\rho_{2}-s} \\
& -\sum_{l=1}^{\sigma-1} g_{l}^{k}(s-l) t^{-l}+0\left(t^{-\sigma}\right) .
\end{aligned}
$$

Proof. For $j=1$, we have

$$
\begin{aligned}
& \sum_{m=0}^{\infty} g_{1}^{k}(m+s) t^{m} \\
&=\sum_{m=0}^{\infty} \frac{\psi\left(\rho_{2}-a_{k k}\right)-\psi\left(\rho_{2}-a_{k k}+m+s+1\right)}{\Gamma\left(m+s+\rho_{2}-a_{k k}+1\right)} \lambda_{k}^{m+s} t^{m} \\
&=\sum_{m=0}^{\infty} \frac{\psi\left(\rho_{2}-a_{k k}+s\right)-\psi\left(\rho_{2}-a_{k k}+s+m+1\right)}{\Gamma\left(m+s+\rho_{2}-a_{k k}+1\right)} \\
& \quad-\sum_{m=1}^{\infty} \frac{\psi\left(\rho_{2}-a_{k k}+s\right)-\psi\left(\rho_{2}-a_{k k}\right)}{\Gamma\left(m+s+\rho_{2}-a_{k k}+1\right)} \lambda_{k}^{s}\left(\lambda_{k} t\right)^{m} \\
&=\lambda_{k}^{s} x_{2}\left(\lambda_{k} t, a_{k k}-\rho_{2}-s\right)-\left(\psi\left(\rho_{2}-a_{k k}+s\right)\right. \\
&\left.\quad-\psi\left(\rho_{2}-a_{k k}\right)\right) \lambda_{k}^{s} x_{1}\left(\lambda_{k} t, \rho_{2}-a_{k k}-s\right)
\end{aligned}
$$




$$
\begin{aligned}
& =\lambda_{k}^{s}\left[\psi\left(\rho_{2}-a_{k k}+s\right)-\log \lambda_{k} t\right] e^{\lambda_{k} t}\left(\lambda_{k} t\right)^{a_{k k}-\rho_{2}-s} \\
& -\lambda_{k}^{s}\left[\psi\left(\rho_{2}-a_{k k}+s\right)-\psi\left(\rho_{2}-a_{k k}\right)\right] e^{\lambda_{k} t}\left(\lambda_{k} t\right)^{a_{k k}-\rho_{2}-s} \\
& -\sum_{l=1}^{\sigma} \frac{\psi\left(\rho_{2}-a_{k k}+s\right)-\psi\left(\rho_{2}-a_{k k}+s-l+1\right)}{\Gamma\left(s-l+\rho_{2}-a_{k k}+1\right)} \lambda_{k}^{s}\left(\lambda_{k} t\right)^{-l} \\
& +\sum_{l=1}^{\sigma} \frac{\psi\left(\rho_{2}-a_{k k}+s\right)-\psi\left(\rho_{2}-a_{k k}\right)}{\Gamma\left(s-l+\rho_{2}-a_{k k}+1\right)} \lambda_{k}^{s}\left(\lambda_{k} t\right)^{-l}+0\left(t^{-\sigma}\right)
\end{aligned}
$$

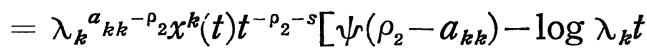

$$
\begin{aligned}
& -\sum_{l=1}^{\sigma-1} \frac{\psi\left(\rho_{2}-a_{k k}\right)-\psi\left(\rho_{2}-a_{k k}+s-l+1\right)}{\Gamma\left(s-l+\rho_{2}-a_{k k}+1\right)} \lambda_{k}^{s} t^{-l}+O\left(t^{-\sigma}\right) \\
& =\lambda_{k}{ }^{a_{k k}}{ }^{-\rho_{2}}\left\{\psi\left(\rho_{2}-a_{k k}\right)-\log \lambda_{k} t\right\} x^{k}(t) t^{-\rho_{2}-s} \\
& -\sum_{l=1}^{\sigma-1} g_{l}^{k}(s-l) t^{-l}+0\left(t^{-\sigma}\right) . \quad\left(t \in \mathfrak{D}_{k}\right) .
\end{aligned}
$$

This proves (2.18), and (2.17) can be proved similarly.

3. Difference systems. In case two eigenvalues $\rho_{1}$ and $\rho_{2}$ of the matrix A satisfy $\rho_{2}-\rho_{1}=m_{0}$, for a positive integer $m_{0}$, there is a fundamental set of solutions of the following form:

$$
\begin{aligned}
& X_{1}(t)=\left(X_{2}(t)\right) \log t+t^{\rho_{1}} \sum_{m=0}^{\infty} G_{1}(m) t^{m} \\
& X_{j}(t)=t^{\rho_{j}} \sum_{m=0}^{\infty} G_{j}(m) t^{m} \quad(j \neq 1)
\end{aligned}
$$

Proposition 3. 1. The coefficient vectors $G_{j}(m)(j=2,3, \cdots, n)$ satisfy

$$
\left(\rho_{j}+m-A\right) G_{j}(m)=B G_{j}(m-1)
$$

with the initial condition

$$
\left(\rho_{j}-A\right) G_{j}(0)=0
$$

and the vector $G_{1}(m)$ satisfies

$$
\left(\rho_{1}+m-A\right) G_{1}(m)=B G_{1}(m-1)-G_{2}\left(m-m_{0}\right)
$$

with the initial condition

$$
\left(\rho_{1}-A\right) G_{1}(0)=0 .
$$

Prof. Obvious from direct computations.

Proposition 3.2. With the pentagonal condition upon the eigen-vrlues 
of $B$, coefficient vectors $H^{k}(s)$ of the formal solutions (1.3) satisfy

$$
\left(a_{k k}-s-A\right) H^{k}(s)=\left(B-\lambda_{k}\right) H^{k}(s+1) \quad(k=1,2, \cdots, n)
$$

with the initial condition

$$
\left(B-\lambda_{k}\right) H^{k}(0)=0 . \quad(k=1,2, \cdots, n)
$$

Proof. Obvious from direct computations.

Definition 3.1. Define formal power series in a complex variable $\varepsilon$ by

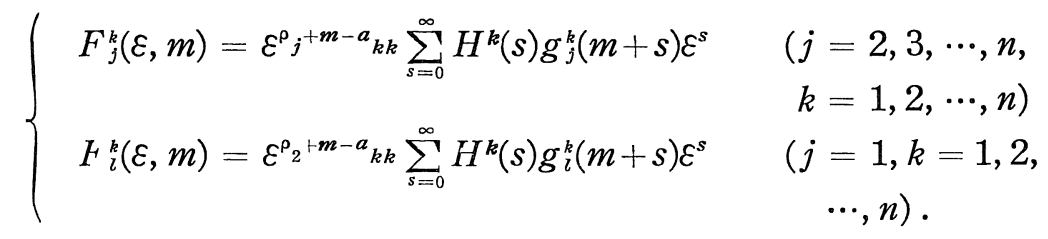

Proposition 3. 3. With the pentagonal condition on $B, F_{j}^{k}(\varepsilon, m)(j \neq 1)$ converge uniformly on the closed unit disk $|\varepsilon| \leqq 1$ of the complex $\varepsilon-$ plane, and $F_{j}^{k}(1, m)(j=2,3, \cdots, n, k=1,2, \cdots, n$.) satisfy (3.3).

Proof. We will show first that $F_{j}^{k}(\varepsilon, m)$ are solutions of

$$
\left\{\varepsilon-\frac{1}{\lambda_{k}}\left(\lambda_{k}-B\right)\right\} \frac{d F_{j}^{k}(\varepsilon, m)}{d \varepsilon}=\left(\rho_{j}+m-A\right) F_{j}^{k}(\varepsilon, m)
$$

From the proposition 2.4, we have formally,

$$
\begin{aligned}
\varepsilon \frac{d F_{j}^{k}(\varepsilon, m)}{d \varepsilon} & =\sum_{s=0}^{\infty}\left(m+s+\rho_{j}-a_{k k}\right) H^{k}(s) g_{j}^{k}(m+s) \varepsilon^{\rho_{i}{ }^{-a}{ }_{k k}+m+s} \\
& =\lambda_{k} \sum_{s=0}^{\infty} g_{j}^{k}(m-1+s) H^{k}(s) \varepsilon^{\rho_{j}-a_{k k^{+m}+s}} \\
& =\lambda_{k} \varepsilon F_{j}^{k}(\varepsilon, m-1)
\end{aligned}
$$

On the other hand, we can use (3.7) and (3.8) to have

$$
\begin{aligned}
\varepsilon \frac{d F_{j}^{k}(\varepsilon, m)}{d \varepsilon}= & \sum_{s=0}^{\infty}\left(s-a_{k k}+A+m+\rho_{j}-A\right) H^{k}(s) g_{j}^{k}(m+s) \varepsilon^{\rho_{j}^{+m-a} k_{k}^{+s}} \\
= & \left(\lambda_{k}-B\right) \sum_{s=0}^{\infty} H^{k}(s) g_{j}^{k}(m-1+s) \varepsilon^{\rho_{j}^{-a}}{ }_{k k^{+m-1+s}} \\
& +\left(B-\lambda_{k}\right) H^{k}(0) g_{j}^{k}(m-1) \varepsilon^{\rho_{j}^{-a}{ }_{k k^{+m-1}}} \\
& \quad+\left(m+\rho_{j}-A\right) F_{j}^{k}(\varepsilon, m) \\
= & \left(\lambda_{k}-B\right) F_{j}^{k}(\varepsilon, m-1)+\left(m+\rho_{j}-A\right) F_{j}^{k}(\varepsilon, m)
\end{aligned}
$$


We have (3.10) formally by eliminating $F_{j}^{k}(\varepsilon, m-1)$.

Each system (3.10), for fixed $j, k$, has $n$ regular singular points in the finite $\varepsilon$-plane, namely those at $\frac{\lambda_{k}-\lambda_{i}}{\lambda_{k}}(i=1,2, \cdots, n)$, and it is easy to see that $F_{j}^{k}(\varepsilon, m)$ is the solution which corresponds to the characteristic exponent $\rho_{j}+m-a_{k k}$ at $\varepsilon=0$. Hence the formal power series in $\varepsilon$ converges uniformly on the closed disk with center at the origin and with radius less than any of the absolute values of $\frac{\lambda_{k}-\lambda_{i}}{\lambda_{k}}(i \neq k)$. But thanks to our pentagonal condition all of them exceed 1. This, also, justifies the formal termwise differentiations above in deriving (3.10)

Let us substitute $\varepsilon=1$ in $F_{\tilde{l}}^{k}(\varepsilon, m)$, and we have

$$
\begin{aligned}
& \left(\rho_{j}+m-A\right) F_{j}^{k}(1, m)-B F_{j}^{k}(1, m-1) \\
= & \left(\rho_{j}+m-A\right) \sum_{s=0}^{\infty} H^{k}(s) g_{j}^{k}(m+s)-B \sum_{s=0}^{\infty} H^{k}(s) g_{j}^{k}(m-1+s) \\
= & \sum_{s=0}^{\infty}\left(\rho_{j}+m+s-a_{k k}+a_{k k}-s-A\right) H^{k}(s) g_{j}^{k}(m+s) \\
& -B \sum_{s=0}^{\infty} H^{k}(s) g_{j}^{k}(m-1+s) \\
= & \lambda_{k} \sum_{s=0}^{\infty} H^{k}(s) g_{j}^{k}(m-1+s)-\left(\lambda_{k}-B\right) \sum_{s=0}^{\infty} H^{k}(s+1) g_{j}^{k}(m+s) \\
& -B \sum_{s=0}^{\infty} H^{k}(s) g_{j}^{k}(m-1+s) \\
= & \left(\lambda_{k}-B\right) H^{k}(0) g_{j}^{k}(m-1)=0
\end{aligned}
$$

Here the convergence of the series such as $\sum_{s=0}^{\infty} s H^{k}(s) g_{j}^{k}(m+s)$ is guaranteed by the convergence of the corresponding power series in $\varepsilon$, say, the derivative of $F_{j}^{k}(\varepsilon, m)$ at $\varepsilon=1$. This completes the proof.

Corollary。 $F_{j}^{k}(1,0)(j=2,3, \cdots, n=1,2, m, n)$ are values at $\varepsilon=1$ of the solution $F_{j}^{k}(\varepsilon, 0)$ of the systems,

$$
\left\{\varepsilon-\frac{1}{\lambda_{k}}\left(\lambda_{k}-B\right)\right\} \frac{d F_{j}^{k}(\varepsilon, 0)}{d \varepsilon}=\left(\rho_{j}-A\right) F_{j}^{k}(\varepsilon, 0)
$$

which correspond to the characteristic exponents $\rho_{j}-a_{k k}$ at $\varepsilon=0$. 
Proposition 3.4. $F_{j}^{k}(\varepsilon, m)(k=1,2, \cdots, n)$ are solutions of the following inhomogeneous systems of differential equations,

$$
\begin{aligned}
\left\{\varepsilon-\frac{1}{\lambda_{k}}\left(\lambda_{k}-B\right)\right\} \frac{d F_{1}^{k}(\varepsilon, m)}{d \varepsilon} & =\left(\rho_{2}+m-A\right) F_{1}^{k}(\varepsilon, m) \\
& -\frac{1}{\varepsilon \lambda_{k}}\left(B-\lambda_{k}\right) F_{2}^{k}(\varepsilon, m) .
\end{aligned}
$$

And the formal series converge uniformly on the closed unit of E-plane.

Proof. By proposition 2.4, and by proposition 3.2., following two relations are derived formally:

$$
\begin{aligned}
& \varepsilon \frac{d F_{1}^{k}(\varepsilon, m)}{d \varepsilon}=\sum_{s=0}^{\infty}\left(\rho_{2}+m+s-a_{k k}\right) H^{k}(s) g_{1}^{k}(m+s) \varepsilon^{\rho_{2}-a_{k k^{+m+s}}} \\
& =\lambda_{k} \varepsilon F_{1}^{k}(\varepsilon, m-1) F_{2}^{k}(\varepsilon, m) \\
& \varepsilon_{-} \frac{d F_{1}^{k}(\varepsilon, m)}{d \varepsilon}=\sum_{s=0}^{\infty}\left\{\left(\rho_{2}+m-A\right)+\left(s-a_{k k}+A\right) H^{k}(s) g_{1}^{k}(m+s) \varepsilon^{o_{2}{ }^{-a}{ }_{k k^{+m+s}}}\right. \\
& =\left(\rho_{2}+m-A\right) F_{1}^{k}(\varepsilon, m)-\left(B-\lambda_{k}\right) F_{1}^{k}(\varepsilon, m-1) \text {. }
\end{aligned}
$$

Elimination of $F_{1}^{k}(\varepsilon, m-1)$ yields (3.12).

The homogeneous part of (3.12) has only $n$ singular points at $\varepsilon=\frac{\lambda_{k}-\lambda_{i}}{\lambda_{k}}(i=1,2, \cdots, n)$, as well as the function $\frac{1}{\varepsilon} F_{2}^{k}(\varepsilon, m)$ for some fixed $k$. Now, owing to the pentagonal condition, the configuration of those singular points other than zero assures the convergence of the formal series.

Proposition 3. 5. $F_{1}^{k}(1, m)(k=1,2, \cdots, n)$ satisfy

$$
\left(\rho_{2}+m-A\right) F_{1}^{k}(1, m)=B F_{1}^{k}(1, m-1)-F_{2}^{k}(1, m) .
$$

Proof. $\quad\left(\rho_{2}+m-A\right) F_{1}^{k}(1, m)-B F_{1}^{k}(1, m-1)$

$$
\begin{aligned}
&=\sum_{s=0}^{\infty}\left\{\left(\rho_{2}+m-s-a_{k k}\right)-\left(s-a_{k k}+A\right) H^{k}(s) g_{1}^{k}(m+s)\right. \\
& \quad-B \sum_{s=0}^{\infty} H^{k}(s) g_{1}^{k}(m-1+s) \\
&=\lambda_{k} \sum_{s=0}^{\infty} H^{k}(s) g_{1}^{k}(m-1+s)-\sum_{s=0}^{\infty} H^{k}(s) g_{2}^{k}(m+s) \\
& \quad-B \sum_{s=0}^{\infty} H^{k}(s) g_{1}^{k}(m-1+s)+\left(B-\lambda_{k}\right) \sum_{s=0}^{\infty} H^{k}(s+1) g_{1}^{k}(m+s)
\end{aligned}
$$




$$
=\sum_{s=0}^{\infty} H^{k}(s) g_{2}^{k}(m+s)-\left(B-\lambda_{k}\right) H^{k}(0) g_{1}^{k}(m-1)=-F_{2}^{k}(1, m)
$$

Definition 3.2. For a positive integer $\sigma$, define vector valued functions $P_{j}^{k}, \sigma(w)(j=2,3, \cdots, n, k=1,2, \cdots, n)$ of a complex variable $w$ by

$$
F_{j}^{k}(1, w)=\sum_{s=0}^{\sigma-1} H^{k}(s) g_{j}^{k}(w+s)+g_{j}^{k}(w+\sigma) P_{j}^{k},{ }_{\sigma}(w)
$$

or equivalently, by

$$
P_{j, \sigma}^{k}(w)=\frac{1}{g_{j}^{k}(w+\sigma)} \sum_{s=\sigma}^{\infty} H^{k}(s) g_{j}^{k}(w+s)
$$

Proposition 3.6. If $h_{0}$ is defind by,

$$
h_{0}=\operatorname{Max}\left\{\frac{3}{2} \Re e\left(\rho_{j}-a_{k k}\right) ; 1 \leqq j \leqq n, 1 \leqq k \leqq n\right\}
$$

then $P_{j, \sigma}^{k}(w)$ are vectors all of whose components are holomorphic and bounded in the right half-plane

$$
\Re e w \geqq h_{0}-\sigma
$$

Proof. It is convenient to introduce a function $q_{j, \sigma}^{k}\left(w, w_{0}, s\right)$ $(\sigma \leq s)$ defined by

$$
\begin{aligned}
q_{j, \sigma}^{k}\left(w, w_{0}, s\right) \\
\quad=\frac{g_{j}^{k}\left(w_{0}+\sigma\right)}{g_{j}^{k}(w+\sigma)}\left\{\frac{g_{j}^{k}(w+s)}{g_{j}^{k}\left(w_{0}+s\right)}-\frac{g_{j}^{k}(w+s-1)}{g_{j}^{k}\left(w_{0}+s-1\right)}\right. \\
=\frac{\Gamma\left(w_{0}+s+\rho_{j}-a_{k k}+1\right)}{\Gamma\left(w_{0}+\sigma+\rho_{j}-a_{k k}+1\right)} \frac{\Gamma\left(w+\sigma+\rho_{j}-a_{k k}+1\right)}{\Gamma\left(w+s+\rho_{j}-a_{k k}+1\right)} \frac{w_{0}-w}{w_{0}+s+\rho_{j}-a_{k k}} \\
=\frac{w-w_{0}}{w+s+\rho_{j}-a_{k k}} \frac{\left(w_{0}-s-1+\rho_{j}-a\right) \cdots\left(w_{0}+\sigma+1+\rho_{j}-a\right)}{\left(w+s-1+\rho_{j}-a_{k k}\right) \cdots\left(w+\sigma+1+\rho_{j}-a_{k k}\right)}
\end{aligned}
$$

Applying Abel's transformation to (3.15), we have

$$
\begin{aligned}
P_{j, \sigma}^{k}(w) & =\frac{1}{g_{j}^{k}\left(w_{0}+\sigma\right)} \sum_{s=\sigma}^{\infty} H^{k}(s) g_{j}^{k}\left(w_{0}+s\right) \frac{g_{j}^{k}\left(w_{0}+\sigma\right)}{g_{j}^{k}(w+\sigma)} \frac{g_{j}^{k}(w+s)}{g_{j}^{k}\left(w_{0}+s\right)} \\
& =P_{j, \sigma}^{k}\left(w_{0}\right)+\frac{1}{g_{j}^{k}\left(w_{0}+\sigma\right)} \sum_{s=\sigma+1}^{\infty} H^{k}(s) g_{j}^{k}\left(w_{0}+s\right) \sum_{p=\sigma+1}^{s} q_{j, \sigma}^{k}\left(w, w_{0}, p\right) \\
& =P_{j, \sigma}^{k}\left(w_{0}\right)+\frac{1}{g_{j}^{k}\left(w_{0}+\sigma\right)} \sum_{p=\sigma+1}^{\infty} q_{j, \sigma}^{k}\left(w, w_{0}, p\right) \sum_{s=p}^{\infty} H^{k}(s) g_{j}^{k}\left(w_{0}+s\right)
\end{aligned}
$$




$$
\begin{aligned}
& =P_{j, \sigma}^{k}\left(w_{0}\right)+\frac{1}{g_{j}^{k}\left(w_{0}+\sigma\right)} \sum_{p=\sigma_{+1}}^{\infty} q_{j, \sigma}^{k}\left(w, w_{0}, p\right) \sum_{s=p}^{\infty} H^{k}(s) g_{j}^{k}\left(w_{0}+s\right) \\
& =P_{j, \sigma}^{k}\left(w_{0}\right)+\frac{1}{g_{j}^{k}\left(w_{0}+\sigma\right)} \sum_{p=\sigma+1}^{\infty}\left[q_{j, \sigma}^{k}\left(w, w_{0}, p\right) g_{j}^{k}\left(w_{0}+p\right) P_{j, \sigma}^{k}\left(w_{0}\right)\right]
\end{aligned}
$$

For the sake of definiteness, let us take

$$
w_{0}=\frac{1}{4}\left(\rho_{j}-a_{k k}\right)-\sigma
$$

then for all $w$ in the half-plane (3.17) and for sufficiently large value of $s$, we have

$$
\begin{aligned}
\left|q_{j, \sigma}^{k}\left(w, w_{0}, s\right)\right| & \leqq \\
& \leqq K(s-\sigma)^{-5 / 4}
\end{aligned}
$$

since for these $w$ we have

$$
\left|w+s+\rho_{j}-a_{k k}\right| \geqq \mathfrak{R e}\left(w+s+\rho_{j}-a_{k k}\right) \geqq s-\sigma+\frac{3}{2}
$$

This shows that $\sum_{s=\sigma}^{\infty} q_{j}^{k}, \sigma\left(w, w_{0}, s\right)$ are absolutely convergent and their sums are uniformly bounded in (3.17). While $g_{j}^{k}\left(w_{0}+p\right) P_{j, \sigma}^{k}\left(w_{0}\right)$ $(p=\sigma+1, \cdots)$, being remainders of absolutely convergent power series $F_{j}^{k}\left(\varepsilon, w_{0}\right)$ at certain point inside the circle of convergence, they are naturally bounded. Thus our proposition is proved by following the well known reasoning of Abel.

Proposition 3.7. For a fixed value of $j(j=2,3, \cdots, n)$ the set $\left\{F_{j}^{k}(1, m) ; k=1,2, \cdots, n\right\}$ constitutes an independent set of solutions of (3.3) for all non-negative integral values of $m$.

Proof. By the preceding proposition, for $w$ such that $\Re e w \geqq h_{0}-1$, we have

$$
\begin{aligned}
F_{j}^{k}(1, w) & \left.=H^{k} 0\right) g_{j}^{k}(w)+g_{j}^{k}(w+1) p_{j, 1}^{k}(w) \\
& =H^{k}(0) g_{j}^{k}(w)\left(1+0\left(\frac{1}{w}\right)\right),
\end{aligned}
$$


or we may write simply

$$
F_{j}^{k}(1, m) \cong H^{k}(0) g_{j}^{k}(m)
$$

for large positive $m$. We use these asymptotic expressions to calculate the Casorati-determinant:

$$
\begin{aligned}
C(w) & =\operatorname{det}\left(F_{j}^{1}, F_{j}^{2}, \cdots, F_{j}^{n}\right) \\
& \cong \operatorname{det}\left(H^{1}(0), H^{2}(0), \cdots, H^{n}(0)\right) g_{j}^{1}(w) \cdots g_{j}^{\prime}(w)
\end{aligned}
$$

Since the vectors $H^{k}(0)(k=1,2, \cdots, n)$ are eigenvectors of the matrix $B$ corresponding to $n$ different eigenvalues respectively, the Casoratideterminant $C(w)$ can not be zero in the distant portion of the positive real axis. On the other hand, reference to the system (3.3) which is satisfied by $F_{j}^{k}(1, w)(k=1,2, \cdots, n)$ shows

$$
\operatorname{det}\left(\rho_{j}+w-A\right) C(w)=\operatorname{det} B \cdot C(w-1)
$$

This proves our proposition because we can deduce $C(w-1) \neq 0$ from $C(w) \neq 0$, as long as $\operatorname{det}\left(\rho_{j}+w-A\right) \neq 0 . \quad$ And $\operatorname{det}\left(\rho_{j}+w-A\right)=0$ for integral $w$, only when $j=2$ and $w=-m_{0}$.

Proposition 3. 8. There are sets of scalar constants $\left\{T_{j}^{k} ; k=1,2, \cdots, n\right\}$ $(j=2,3, \cdots, n)$ such that

$$
G_{j}(m)=\sum_{k=1}^{n} T_{j}^{k} F_{j}^{k}(1, m)
$$

are the solutions of the systems (3.3) with the initial conditions (3.4) respectively.

We shall call these sets $\left\{T_{j}^{k} ; k=1,2, \cdots, n\right\}(j=2,3, \cdots, n)$ the sets of $S$-multipliers for respective solutions $G_{j}(m)$. They are determined by the systems of equations

$$
G_{j}(0)=\sum_{k=1}^{n} T_{j}^{k} F_{j}^{k}(1,0) .
$$

What is most important here is the fact that $F_{j}^{k}(1,0)(j=2,3, \cdots, n$; $k=1,2, \cdots, n)$ are all expressed in terms of convergent series. It we drop the pentagonal condition, they are, in general, divergent expressions, and some modifications of the theory are necessary. One of such modifications was carried out in our preceding paper. 
Proposition 3.9. With the help of $S$-multiplies for $G_{2}(m)$, the vector

$$
G_{1}^{*}(m)=\sum_{k=1}^{n} T_{2}^{k} F_{1}^{k}\left(1, m-m_{0}\right)
$$

satisfies the difference system (3.5).

Proof. Since the vectors $F_{1}^{k}(1, m)$ satisfy (3.13), we have

$$
\left(m+\rho_{1}-A\right) F_{1}^{k}\left(1, m-m_{0}\right)=B F_{1}^{k}\left(1, m-m_{0}-1\right)-F_{2}^{k}\left(1, m-m_{0}\right)
$$

for each $k$. Multiplying $T_{2}^{k}$ to each of these systems, and summing them up on the set of indices $k=1,2, \cdots, n$, we have (3.5)

Proposition 3.10. There is a set of constants $T_{1}^{k} ; k=1,2, \cdots, n$ such that

$$
G_{1}(m)=G_{1}^{*}(m)+\sum_{k=1}^{n} T_{1}^{k} F_{2}^{k}\left(1, m-m_{0}\right)
$$

is the solution of (3.5) with the initial condition (3.6). They are determined by

$$
G_{1}\left(m_{0}\right)=G_{1}^{*}\left(m_{0}\right)+\sum_{k=1}^{n} T_{1}^{k} F_{2}^{k}(1,0)
$$

Proof. Since the system (3.5) is inhomogeneous, a general solution is obtained by adding a general solution of the homogeneous part to a special solution. And we know that $G_{1}^{*}(m)$ is a special solution of (3.5) by the preceding proposition. Solvability of the system (3.23) is assured by the independence of the set $\left\{F_{2}^{k}(1, m)\right.$; $k=1,2, \cdots, n\}$

\section{Asymptotic expansions of $X_{j}(t)(j=2,3, \cdots, n$.)}

Proposition 4. 1. (E.M. Wright) If $\varphi(w)$ is holomorphic and bounded in the right half-plane

$$
\Re \mathrm{e} w \geqq h^{\prime}>0
$$

and

$$
h^{\prime}>\frac{3}{2}-R e \beta
$$

then we have 


$$
\sum_{m=\left\{h^{\prime}\right\}+1}^{\infty} \frac{\varphi(m)}{\Gamma(m+\beta)} z^{m}=0\left(e^{z} z^{1-\beta}\right)+0\left(z^{h^{\prime}}\right)
$$

as $z$ tends to infinity in the sector

$$
|\arg z| \leqq \frac{3}{2} \pi .
$$

Proposition 4.2. If $h_{0}$, defined by (3.16), is positive, aud

$$
\sigma>h=\left[h_{0}\right]+1,
$$

we have

$$
\begin{aligned}
\sum_{m=0}^{\infty} g_{j}^{k}(m+\sigma) & P_{j}^{k}{ }_{j}(m) t^{m}=0\left(e^{\lambda k^{t}} t^{a}{ }_{k k^{-\rho_{j}}-\sigma}\right) \\
& -\sum_{l=1}^{\sigma-k} g_{j}^{k}(\sigma-l) P_{j, \sigma}^{k}(-l) t^{-l}+0\left(t^{h_{0}^{-\sigma}}\right)
\end{aligned}
$$

in the sector

$$
\left|\arg \lambda_{k} t\right| \leqq \frac{3}{2} \pi
$$

Proof. We decompose the sum in the left side of (4.5) as follows.

$$
\begin{aligned}
& \sum_{m=0}^{\infty} g_{j}^{k}(m+\sigma) P_{j, \sigma}^{k}(m) t^{m} \\
&=\sum_{m=h-\sigma}^{\infty} g_{j}^{k}(m+\sigma) P_{j, \sigma}^{k}(m) t^{m}-\sum_{l=1}^{\sigma-h} g_{i}^{k}(\sigma-l) P_{j, \sigma}^{k}(-l) t^{-l} \\
&=\sum_{m=h}^{\infty} g_{j}^{k}(m) P_{j, \sigma}^{k}(m-\sigma) t^{m-\sigma}-\sum_{l=1}^{\sigma-h} g_{j}^{k}(\sigma-l) P_{j, \sigma}^{k}(-l) t^{-l} \\
&= t^{-\sigma} \sum_{\infty}^{m=h} \frac{P_{j, \sigma}^{k}(m-\sigma)}{\Gamma\left(m+\rho_{j}-a_{k k}+1\right)}\left(\lambda_{k} t\right)^{m} \\
& \quad-\sum_{l=1}^{\sigma-h} g_{j}^{k}(\sigma-l) P_{j, \sigma}^{k}(-l) t^{-l} .
\end{aligned}
$$

We observe, with the help of proposition 3.6., that each component of $P_{j, \sigma}^{k}(w-\sigma)$ is holomorphic and bounded in the right half-plane

$$
\Re e w \geqq h_{0}>0 \text {. }
$$

The condition (4.2) of the foregoing proposition is now replaced by

$$
h_{0}>\frac{3}{2}-\Re e\left(\rho_{j}-a_{k k}\right)-1
$$


and is automatically satisfied by the definition (3.16) of $h_{0}$. Now we apply proposition 4.1 to each component of

$$
\sum_{m=k}^{\infty} \frac{P_{j, \sigma}^{k}(m-\sigma)}{\Gamma\left(m+\rho_{j}-a_{k k}+1\right)}\left(\lambda_{k} t\right)^{m}
$$

to have present proposition.

Corollary. If $h_{0}$ is not positive, take any arbitrarily small positive real $h_{0}^{*}$ and we have

$$
\begin{aligned}
\sum_{m=0}^{\infty} g_{j}^{k}( & +\sigma) P_{j, \sigma}^{k}(m) t^{m}=0\left(e^{\lambda} k^{t} t^{a}{ }_{k k^{-\rho}}{ }^{-\sigma}\right) \\
& -\sum_{l=1}^{\sigma-1} g_{j}^{k}(\sigma-l) P_{j, \sigma}^{k}(-l) t^{-l}+0\left(t^{h_{0}^{*}-\sigma}\right)
\end{aligned}
$$

Proof. Since $P_{j, \sigma}^{k}(w-\sigma)$ is holomorphic and bounded in

$$
\Re \mathrm{e} w \geqq h_{0}^{*}>h_{0}
$$

and

$$
h>h_{0}>\frac{3}{2}-\Re e\left(\rho_{j}-a_{k k}\right)-1
$$

we have the corollary.

Although the case $h_{0} \leq 0$ may happen according to the form of the matrix $A$, we only treat the case $h_{0}>0$ to avoid complicated statements in the following.

Proposition 4. 3. In the sector $\mathfrak{D}_{k}$ defined by (2.16), we have

$$
\begin{aligned}
\sum_{m=0}^{\infty} F_{j}^{k}(1, m) t^{m}= & \lambda_{k}{ }^{a}{ }_{k k^{-\rho}}{ }_{j} e^{\lambda} k^{t} t^{a}{ }_{k k^{-\rho}} j\left\{\sum_{s=0}^{\sigma-1} H^{k}(s) t^{-s}+0\left(t^{-\sigma}\right)\right. \\
& -\sum_{l=1}^{\sigma-h} F_{j}^{k}(1,-l) t^{-l}+0\left(t^{h-\sigma}\right)
\end{aligned}
$$

for $j=2,3, \cdots, n, k=1,2, \cdots, n$.

Proof. By definition 3.2., we have

$$
\begin{aligned}
\sum_{m=0}^{\infty} F_{j}^{k}(1, m) t^{m} & =\sum_{m=0}^{\infty}\left[\sum_{s=0}^{\sigma-1} H^{k}(s) g_{j}^{k}(m+s)+g_{j}^{k}(m+\sigma) P_{j, \sigma}^{k}(m)\right] t^{m} \\
& =\sum_{s=0}^{\sigma-1} H^{k}(s) \sum_{m=0}^{\infty} g_{j}^{k}(m+s) t^{m}+\sum_{m=0}^{\infty} g_{j}^{k}(m+\sigma) P_{j, \sigma}^{k}(m) t^{m}
\end{aligned}
$$


Applying proposition 2.5, to the first term, we have

$$
\begin{aligned}
\sum_{s=0}^{\sigma-1} H^{k}(s) \sum_{m=0}^{\infty} g_{j}^{k}(m+s) t^{m}= & \sum_{s=0}^{\sigma-1} H^{k}(s)\left[\lambda_{k}{ }^{a}{ }_{k k^{-\rho}} j e^{\lambda_{k} t} t^{a}{ }_{k k^{-\rho}} j^{-s}\right. \\
& \left.-\sum_{l=1}^{\sigma-h} g_{j}^{k}(s-l) t^{-l}+0\left(t^{h-\sigma}\right)\right] \\
= & \lambda_{k}{ }^{a} k k^{-\rho_{j}} e^{\lambda_{k}}{ }^{t} t^{a}{ }_{k k^{-\rho_{j}}} \sum_{s=0}^{\sigma-1} H^{k}(s) t^{-s} \\
& -\sum_{l=1}^{\sigma-1} \sum_{s=0}^{\sigma-h} g_{j}^{k}(s-l) H^{k}(s) t^{-l}+0\left(t^{h-\sigma}\right)
\end{aligned}
$$

Adding (4.5), we have

$$
\begin{aligned}
\sum_{m=0}^{\infty} F_{j}^{k}(1, m) t^{m}= & \lambda_{k}{ }^{{ }^{a}{ }_{k k^{-}}{ }^{-\rho}} j e^{\lambda_{k}} t^{t} t_{k k^{-}}{ }^{-\rho}\left\{\sum_{s=0}^{\sigma-1} H^{k}(s) t^{-s}+0\left(t^{-\sigma}\right)\right\} \\
& -\sum_{l=1}^{\sigma-1}\left\{\sum_{s=0}^{\sigma-h} g_{j}^{k}(s-l) H^{k}(s)+g_{j}^{k}(\sigma-l) P_{j, \sigma}^{k}(-l) t^{-l}\right. \\
& +0\left(t^{h-\sigma}\right)
\end{aligned}
$$

We have the proposition because of the definition 3.2. for $w=-l$ :

$$
\sum_{s=0}^{\sigma-1} g_{j}^{k}(s-l) H^{k}(s)+g_{j}^{k}(\sigma-l) P_{j, \sigma}^{k}(-l)=F_{j}^{k}(1,-l) .
$$

Theorem 4.1. For $j=2,3, \cdots, n$, we have

$$
\begin{aligned}
X_{j}(t)= & \sum_{k=1}^{n} T_{j}^{k} \lambda_{k}{ }_{k k k^{-\rho}} e^{\lambda k^{t}} t^{a}{ }_{k k}\left\{\sum_{s=0}^{\sigma-1} H^{k}(s) t^{-s}+0\left(t^{-\sigma}\right)\right\} \\
& +0\left(t^{h-\sigma+\rho_{j}}\right)
\end{aligned}
$$

in the sector

$$
\mathfrak{D}=\bigcap_{k=1}^{\infty} \mathfrak{D}_{k}
$$

Proof. By proposition 3.9., we multiply $t_{j}^{\rho} T_{j}^{k}$ to (4.7) for each $k$, and sum over $k=1,2, \cdots, n$, to have

$$
\begin{aligned}
X_{j}(t)= & t^{\rho_{j}} \sum_{k=1}^{n} T_{j}^{k} \sum_{m=0}^{\infty} F_{j}^{k}(1, m) t^{m} \\
= & \sum_{k=1}^{n} T_{j}^{k} \lambda_{k}{ }_{k k{ }^{-\rho}} e^{\lambda}{ }^{\lambda}{ }^{t} t^{a}{ }_{k k}\left\{\sum_{s=0}^{\sigma-1} H^{k}(s) t^{-s}+0\left(t^{-\sigma}\right)\right\} \\
& -\sum_{k=1}^{n} T_{j}^{k} \sum_{l=1}^{\sigma-h} F_{j}^{k}(1,-l) t^{-l+{ }_{j}}+0\left(t^{h-\sigma+\rho_{j}}\right)
\end{aligned}
$$

Applying the proposition 3.9. again to the second term of the above expression, we have 


$$
\begin{aligned}
\sum_{k=1}^{n} T_{j}^{k} \sum_{l=1}^{\sigma-h} F_{j}^{k}(1,-l) t^{-l} & =\sum_{l=1}^{\sigma-h} \sum_{k=1}^{n} T_{j}^{k} F_{j}^{k}(1,-l) t^{-\imath} \\
& =\sum_{l=1}^{\sigma-h} G_{j}(-l) t^{-l}=0
\end{aligned}
$$

because of the initial condition (3.4) for $G_{j}(m)$.

Corollary. If, in the sector $\mathfrak{D}$, there is at least one index $k$, for which $\Re \mathrm{e}\left(\lambda_{k} t\right)$ is non-negative, then we have

$$
X_{j}(t) \cong \sum_{k=1}^{n} T_{j}^{k} \lambda_{k}{ }^{a_{k k}-{ }^{-\rho} j x^{k}}(t)
$$

Remark. The exact meaning of these asymptotic relations can be seen from the exact expressions in theorem 4.1., namely, if there is some $k$, for which $\Re \mathrm{e} \lambda_{k} t \geqq 0$ holds, then

$$
X_{j}(t) \cong \sum_{k} \lambda_{k}{ }^{a}{ }_{k k^{-}}{ }_{j} T_{j}^{k} X^{k}(t) \quad(j=2,3, \cdots, n)
$$

the summation is taken only over those values of $k$ for which $\Re e \lambda_{k} t \geqq 0$. If there is no such $k$, then we must write

$$
X_{j}(t) \cong 0
$$

But even in this case, the right-hand side is asymptotically zero only in comparison with negative powers of $t$, but not with exponentially decreasing (subdominant) solutions.

However, for the sake of simplicity, we shall use the conventional notation

$$
X_{j}(t) \cong \sum_{k=1}^{n} \lambda_{k}{ }^{a_{k k}-\rho_{j}} T_{j}^{k} X^{k}(t)
$$

including such subdominant solutions with the phrase "in the sense of this remark".

Theorem 4. 2. (Continuations to different sectors.) If we denote by the sector

$$
\overline{\mathfrak{D}}_{k}=\left\{t ; \lambda_{k} e^{2 \pi i t} \in \mathfrak{D}^{*}\right\} \quad(k=p+1, p+2, \cdots, n)
$$

we have

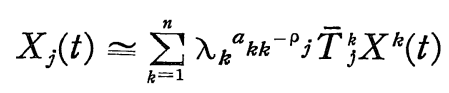


in

$$
\overline{\mathfrak{D}}=\left(\bigcap_{k=1}^{p} \mathfrak{D}_{k}\right) \cap\left(\bigcap_{k=p+1}^{n} \overline{\mathfrak{D}}_{k}\right)
$$

where $\bar{T}_{j}^{k}(j=2,3, \cdots, n, k=1,2, \cdots, n)$ are defined by

$$
\begin{cases}\bar{T}_{j}^{k}=T_{j}^{k} & (k=1,2, \cdots, p) \\ \bar{T}_{j}^{k}=e^{2 \pi_{i}\left(a_{\left.k k^{-\rho}\right)}\right)} T_{j}^{k} & (k=p+1, p+2, \cdots, n) .\end{cases}
$$

Asymptotic relations are valid in the sense of the above remark.

Proof. We just changed the arguments of $\lambda_{k}$ 's by $2 \pi$ for $k=p+1, p+2, \cdots, n$.

\section{Asymptotic expansion $X_{1}(t)$}

Proposition 5.1. For $k=1,2, \cdots, n$, series

$$
\frac{1}{g_{2}^{k}(w+\sigma+1)} \sum_{p=\sigma}^{\infty} \frac{g_{2}^{k}(w+p-1)}{w+\rho_{2}-a^{k k}+p+1}
$$

are absolutely convergent and unfformly bounded in the right halfplane (3. 17).

Proof. The $(p-\sigma+1)$-th terms of (5.1) are of the form

$$
\lambda_{k}^{p-\sigma} \frac{1}{w+p+1+\rho_{2}-a_{k k}} \cdot \frac{1}{\left(w+p+1+\rho_{2}-a_{k k}\right) \cdots\left(w+\sigma+2+\rho_{2}-a_{k k}\right)}
$$

which are less than

$$
\left|\lambda_{k}\right|^{p-\sigma} \frac{1}{\left.\left(\frac{5}{2}+p-\sigma\right)\right)} \cdot \frac{\Gamma\left(\frac{7}{2}\right)}{\Gamma\left(\frac{7}{2}+p-\sigma\right)}
$$

in absolute value, because we have

$$
\begin{aligned}
\frac{1}{w+p+\rho_{2}-a_{k k}} & \leqq \frac{1}{\Re e\left(w+p+\rho_{2}-a_{k k}\right)} \leqq \frac{1}{h_{0}-\sigma+\Re e\left(\rho_{2}-a_{k k}\right)+p} \\
& \leqq \frac{1}{p-\sigma+\frac{3}{2}}(p>\sigma)
\end{aligned}
$$

by the definition (3.16) of $h_{0}$. 
Proposition 5. 2. If we define $P_{1, \sigma}^{k}(w)$ by

$$
F_{1}^{k}(1, w)=\sum_{s=0}^{1-\sigma} H^{k}(s) g_{1}^{k}(w+s)+g_{2}^{k}(w+\sigma-1) \cdot P_{1, \sigma}^{k}(w)
$$

or equivalently by

$$
P_{1, \sigma}^{k}(w)=\frac{1}{g_{2}^{k}(w+\sigma-1)} \sum_{s=\sigma}^{\infty} H^{k}(s) g_{1}^{k}(w+s)
$$

then $P_{1, \sigma}^{k}(w)(k=1,2, \cdots, n)$ are uniformly bounded in the right halfplane (3.17).

Proof. We have

$$
\begin{aligned}
\sum_{s=\sigma}^{\infty} H^{k}(s) g_{1}^{k}(w+s)= & \sum_{s=\sigma}^{\infty} H^{k}(s) g_{2}^{k}(w+s)\left[\sum _ { p = \sigma } ^ { s - 1 } \left\{\psi^{k}(w+p+1)\right.\right. \\
& \left.\left.-\psi^{k}(w+p)\right\}+\psi^{k}(w+\sigma)\right]
\end{aligned}
$$

where $\psi^{k}(w)$ are defined by

$$
\psi^{k}(w)=\psi\left(\rho_{2}-a_{k k}\right)-\psi\left(w+\rho_{2}-a_{k k}+1\right) .
$$

We decompose these series as follows.

$$
\begin{aligned}
\psi^{k}(w+\sigma) & \sum_{s=\sigma}^{\infty} H^{k}(s) g_{2}^{k}(w+s) \\
& +\sum_{p=\sigma}^{\infty}\left\{\psi^{k}(w+p+1)-\psi^{k}(w+p)\right\} \sum_{s=p+1}^{\infty} H^{k}(s) g_{2}^{k}(w+s) \\
= & \psi^{k}(w+\sigma) g_{2}^{k}(w+\sigma) P_{2, \sigma}^{k}(w) \\
& \quad-\sum_{p=\sigma}^{\infty} \frac{1}{w+\rho_{2}-a_{k k}+p+1} g_{2}^{k}(w+p+1) P_{2, p^{+1}}^{k}(w) \\
= & g_{2}^{k}(w+\sigma)\left[\psi\left(\rho_{2}-a_{k k}\right)-\psi\left(w+\sigma+\rho_{2}-r_{k k}+1\right)\right] P_{2, \sigma}^{k}(w) \\
& \quad-g_{2}^{k}(w+\sigma+1) \sum_{p=\sigma}^{\infty}\left\{\frac{1}{w+\rho_{2}-a_{k k}+p+1} \frac{g_{2}^{k}(w+p+1)}{g_{2}^{k}(w+\sigma-1)}\right\} P_{2, p^{+1}}^{k}(w) .
\end{aligned}
$$

The second summation of the last sentence is easily seen to be absolutely convergent because of the foregoing proposition and the uniform boundedness of $P_{2, p}^{k}(w)$ in the right half-plane (3.17) for $\sigma \leq p$. And we see, at the same time, that each component of the expression is less than

$$
K_{1}\left|g_{2}^{k}(w+\sigma)\left[\psi\left(\rho_{2}-a_{k k}\right)-\psi\left(w+\sigma+\rho_{2}-a_{k k}+1\right)\right]\right|+K_{2}\left|g_{2}^{k}(w+\sigma+1)\right|
$$


in absolute value. To complete the proof, we have only to use the the estimate of di-gamma function

$$
\psi(z) \simeq \log z
$$

Proposition 5. 3. We have for $k=1,2, \cdots, n$,

$$
\begin{array}{r}
\sum_{m=0}^{\infty} g_{2}^{k}(m+\sigma-1) P_{1, \sigma}^{k}(m) t^{m}=0\left(e^{\lambda}{ }_{k} t t^{a}{ }_{k k^{-}}{ }^{2}{ }^{+1-\sigma}\right) \\
-0\left(t^{h_{0}-\sigma}\right)-\sum_{l=1}^{\sigma-k} g_{2}^{k}(\sigma-1-l) P_{1, \sigma}^{k}(-l) t^{-\iota}
\end{array}
$$

in (4.6) which obviously contains $\mathfrak{D}_{k}$.

Proof. As in the proof of the proposition 4.2., we transform the summation into the form

$$
\begin{aligned}
\sum_{m=0}^{\infty} g_{2}^{k}(m & +\sigma-1) P_{1, \sigma}^{k}(m) t^{m}=\sum_{m=h-\sigma}^{\infty} g_{2}^{k}(m+\sigma-1) P_{1, \sigma}^{k}(m) t^{m} \\
& -\sum_{l=1}^{\sigma-k} g_{2}^{k}(\sigma-1-l) P_{1, \sigma}^{k}(-l) t^{-l} \\
& =t^{-\sigma} \sum_{m=h}^{\infty} \frac{\lambda_{k}^{-1} \cdot P_{1, \sigma}^{k}(m-\sigma)}{\Gamma\left(m+\rho_{2}-a_{k k}\right)}\left(\lambda_{k} t\right)^{m}-\sum_{l=1}^{\sigma-k} g_{2}^{k}(\sigma-1-l) P_{1, \sigma}^{k}(-l) t^{-l}
\end{aligned}
$$

We observe that each component of $\lambda_{k}^{-1} \cdot P_{1, \sigma}^{k}(w-\sigma)$ is uniformly bounded in $\Re e w \geqq h_{0}$ by the preceding proposition, and that the condition (4.2) of the proposition 4.1. is satisfied by the assumption that $m_{0}$ is a positive integer. Indeed, we have

$$
\begin{aligned}
h_{0} & =\operatorname{Max}\left\{\frac{3}{2}-\Re e\left(\rho_{j}-a_{k k}\right)\right\} \geqq \frac{3}{2} \Re e\left(\rho_{1}-a_{k k}\right) \\
& =\frac{3}{2}+m_{0}-\Re e\left(\rho_{2}-a_{k k}\right)>\frac{3}{2}-\Re e\left(\rho_{2}-a_{k k}\right) .
\end{aligned}
$$

Thus we can apply the proposition 4.1. to the first term to obtain

$$
t^{-\sigma} \sum_{m=h}^{\infty} \frac{\lambda_{k}^{-1} \cdot p_{1, \sigma}^{k}(m-\sigma)}{\Gamma\left(m+\rho_{2}-a_{k k}\right)}\left(\lambda_{k} t\right)^{m}=0\left(e^{\lambda_{k}} t^{a}{ }_{k k^{-\rho_{2}+1-\sigma}}\right)+0\left(t^{h_{0}-\sigma}\right) .
$$

This proves our proposition.

Proposition 5.4. For $t$ in $\mathfrak{D}_{k}$, we have 
(5. 5)

$$
\begin{aligned}
\sum_{m=0}^{\infty} F_{1}^{k}(1, m) t^{m} & \\
= & \lambda_{k}^{d}{ }_{k k}{ }^{-\rho_{2} e_{k}{ }_{k} t} t^{a}{ }_{k k}{ }^{-\rho_{2}}\left\{\psi\left(\rho_{2}-a_{k k}\right)-\log \lambda_{k} t\right\} \sum_{s=0}^{\sigma-1} H^{k}(s) t^{-s} \\
& \quad-\sum_{l=1}^{\sigma-h} F_{1}^{k}(1,-l) t^{-l}+0\left(t^{h-\sigma}\right)+0\left(e^{\lambda_{k} t} t^{a}{ }_{k k^{-\rho_{2}+1-\sigma}}\right)
\end{aligned}
$$

for $k=1,2, \cdots, n$.

Proof. By proposition 2.5., we have

$$
\begin{aligned}
& \sum_{m=0}^{\infty} \sum_{s=0}^{\sigma-1} H^{l}(s) g_{1}^{k}(m+s) t^{m}
\end{aligned}
$$

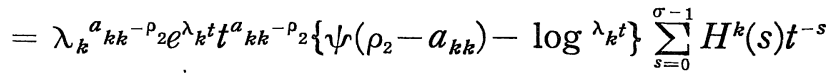

$$
\begin{aligned}
& -\sum_{l=1}^{\sigma-1} t^{-l} \sum_{s=0}^{\sigma-h} H^{k}(s) g_{1}^{k}(s-l)+0\left(t^{h-\sigma}\right) \text {. }
\end{aligned}
$$

Using the definition (5.2) of $P_{1, \sigma}^{k}(w)$ for $w=-l$, we have

$$
\begin{aligned}
& \sum_{l=1}^{\sigma-h} \sum_{s=0}^{\sigma-1} H^{k}(s) g_{1}^{k}(s-l) t^{-l}+\sum_{l=1}^{\sigma-h} g_{2}^{k}(\sigma-1-l) P_{1, \sigma}^{k}(-l) t^{-l} \\
& \quad \sum_{l=1}^{\sigma-h}\left\{\sum_{s=0}^{\sigma-1} H^{k}(s) g_{1}^{k}(s-l)+g_{2}^{k}\left(\sigma^{-1-l}\right) P_{1, \sigma}^{k}(-l)\right\} t^{-l}=\sum_{l=1}^{\sigma-h} F_{1}^{k}(1,-l) t^{-l}
\end{aligned}
$$

We use this identity, and add (5.4) to both sides of (5.6) to have (5.5).

Corollary. We have in $\mathfrak{D}_{k}$, for $k=1,2, \cdots, n$,

$$
\begin{aligned}
\sum_{m=0}^{\infty} F_{1}^{k}\left(1, m-m_{0}\right) t^{m}= & \lambda_{k}{ }^{a_{k k^{-}}{ }_{2}}\left(\psi\left(\rho_{2}-a_{k k}\right)\right. \\
& \left.-\log \lambda_{k} t\right) e^{\lambda_{k} t} t^{a}{ }_{k k}{ }^{-\rho_{1}} \sum_{s=0}^{\infty} H^{k}(s) t^{-s} \\
& -\sum_{l=1}^{\infty} F_{1}^{k}\left(1, m_{0}-l\right) t^{-l}
\end{aligned}
$$

in the sense of the remark of the last section.

Proof.

$$
\begin{aligned}
& \sum_{m=0}^{\infty} F_{1}^{k}\left(1, m-m_{0}\right) t^{m}=\sum_{m=0}^{m_{0}-1} F_{1}^{k}\left(1, m-m_{0}\right) t^{m}+t^{m_{0}} \sum_{m=0}^{\infty} F_{1}^{k}(1, m) t^{m} \\
& \simeq \lambda_{k}{ }^{a_{k k}-\rho_{2}}\left(\psi\left(\rho_{2}-a_{k k}\right)-\log \lambda_{k} t\right) e^{\lambda_{k} t} t^{a_{k k}-\left(\rho_{2}-m_{0}\right)} \sum_{s=0}^{\infty} H^{k}(s) t^{-s}
\end{aligned}
$$




$$
\begin{gathered}
+\sum_{l=1-m_{0}}^{0} F_{1}^{k}\left(1,-l-m_{0}\right) t^{-l}-\sum_{l=1}^{\infty} F_{1}^{k}(1,-l) t^{m_{0}-l} \\
\simeq \lambda_{k}{ }_{k k^{-\rho_{2}}}\left[\psi\left(\rho_{2}-a_{k k}\right)-\log \lambda_{k} t\right] e^{\lambda} k^{t} t^{a} k^{-\rho_{1}} \sum_{s=0}^{\infty} H^{k}(s) t^{-s} \\
+\sum_{l=1-m_{0}}^{0} F_{l}^{k}\left(1,-l-m_{0}\right) t^{-l}-\sum_{l=1-m_{0}}^{\infty} F_{1}^{k}\left(1, m_{0}-l\right) t^{-l}
\end{gathered}
$$

Proposition 5. 5. For $t$ in $\bigcap_{k=1}^{n} \mathfrak{D}_{k}=\mathfrak{D}$, we have

$$
\begin{aligned}
t^{\rho_{1}} \sum_{m=0}^{\infty} G_{1}(m) t^{m} \cong & \sum_{k=1}^{n} \lambda_{k}^{a_{k k^{-\rho}}}\left[T_{1}^{k}+T_{2}^{k}\left\{\psi\left(\rho_{2}-a_{k k}\right)-\log \lambda_{k}\right\}\right] X^{k}(t) \\
& -\left[\sum_{k=1}^{n} \lambda_{k}{ }^{a}{ }_{k k^{-\rho_{2}}} T_{2}^{k} X^{k}(t)\right] \log t
\end{aligned}
$$

in the sense of the remark of the last section.

Proof. By proposition 3.11., and by definition (3.21) of $G_{1}^{*}(m)$, we have

$$
G_{1}(m)=\sum_{k=1}^{n} T_{2}^{k} F_{1}^{k}\left(1, m-m_{0}\right)+\sum_{k=1}^{n} T_{1}^{k} F_{2}^{k}\left(1, m-m_{0}\right)
$$

We first compute the contributions from $F_{2}^{k}\left(1, m-m_{0}\right)(k=1,2, \cdots, n)$ as follows.

$$
\begin{gathered}
t^{\rho_{1}} \sum_{m=0}^{\infty} F_{2}^{k}\left(1, m-m_{0}\right) t^{m}=t^{\rho_{1}} \sum_{m=0}^{m_{0}-1} F_{2}^{k}\left(1, m-m_{0}\right) t^{m}+t^{\rho_{2}} \sum_{m=1}^{\infty} F_{2}^{k}(1, m) t^{m} \\
\simeq \lambda_{k}{ }^{a} k k^{-\rho_{2}} X^{k}(t)-\sum_{l=1}^{\infty} F_{2}^{k}(1,-l) t^{\rho_{2}-l}+\sum_{l=1}^{m_{0}-1} F_{2}^{k}(1,-l) t^{\rho_{2}-l} \\
\simeq \lambda_{k}{ }^{a} k k^{-\rho_{2}} X^{k}(t)-\sum_{l=1}^{\infty} F_{2}^{k}\left(1,-m_{0}-l\right) t^{\rho_{1}-l} .
\end{gathered}
$$

Then we use the preceding corollary to obtain the desired expression, since

$$
\sum_{k=1}^{n} T_{1}^{k} F_{2}^{k}\left(1,-m_{0}-l\right)+\sum_{k=1}^{n} T_{2}^{k} F_{1}^{k}\left(1, m_{0}-l\right)=G_{1}(-l)=0
$$

for positive integral value of $l$.

Theorem 5.2. In the sector $\mathfrak{D}=\bigcap_{k=1}^{n} \mathfrak{D}_{k}$, we have

$$
X_{1}(t) \cong \sum_{k=1}^{n} \lambda_{k}^{a k k^{-\rho_{2}}}\left[T_{1}^{k}+T_{2}^{k}\left\{\psi\left(\rho_{2}-a_{k k}\right)-\log _{k} \lambda_{k}\right\} X^{k}(t)\right.
$$

in the sense of the remark of the last section. 
Proof. We are looking for the asymptotic expresion for

$$
X_{1}(t)=X_{2}(t) \log t+t^{\rho_{1}} \sum_{m=0}^{\infty} G_{1}(m) t^{m}
$$

when $X_{2}(t)$ has the form

$$
X_{2}(t) \cong \sum_{k=1}^{n} \lambda_{k}{ }^{a}{ }_{k k}{ }^{-\rho_{2}} T_{2}^{k} X^{k}(t) \quad(t \in \mathfrak{D})
$$

by the corollary to the theorem 4.1.. Our theorem is proved by the use of (5.8)

6. System of rank 2. Let us consider a system with an irregular singular point of rank 2 :

$$
t \frac{d X}{d t}=\left(A_{0}+t A_{1}+t^{2} A_{2}\right) X
$$

There exists a fundamental set of solutions with convergent expression :

$$
X_{j}(t)=t^{\rho} \sum_{m=0}^{n} G_{j}(m) t^{m}
$$

where coefficients are determined from

$$
\left(\rho_{j}+m\right) G_{j}(m)=A_{0} G_{j}(m)+A_{1} G_{j}(m-1)+A_{2} G_{j}(m-2)
$$

and

$$
\left(\rho_{j}-A\right) G_{j}(0)=0 .
$$

Of course, $\rho_{1}, \cdots, \rho_{n}$ are eigenvalues of $A_{0}$, and we assume no pair of them is congruent modulo integer.

Another set of formal solutions are determined in the form

$$
X^{k}(t) \sim x^{k}(t) \sum_{s=0}^{\infty} H^{k}(s) t^{-s}
$$

by

$$
x^{k}(t)=\exp \left(\frac{\lambda k}{2} t^{2}+\mu_{k} t\right) t^{\sigma}{ }_{k}
$$

and

(6. 7) $\quad\left(\lambda_{k}-A_{2}\right) H^{k}(s+2)+\left(\mu_{k}-A_{1}\right) H^{k}(s+1)+\left(\sigma_{k}-A_{0}\right) H^{k}(s)=s H^{k}(s)$ 
where we assumed $A_{2}=\operatorname{diag}\left(\lambda_{1}, \cdots, \lambda_{n}\right)$ and these eigenvalues satisfy the pentagonal condition.

We define $x_{j}^{k}(t)$ to be the holomorphic solution at the origin of

$$
t \frac{d x_{j}^{k}}{d t}=\left(\sigma_{k}-\rho_{j}+\mu_{k} t+\lambda_{k} t^{2}\right) x_{j}^{k}+c_{j}^{k} \quad\left(c_{j}^{k} ; \text { constant }\right)
$$

If we write

$$
x_{j}^{k}(t)=\sum_{m=0}^{\infty} g_{i}^{k}(m) t^{m}
$$

then $g_{j}^{k}(m)$ satisfy

$$
\left(\rho_{j}+m\right) g_{j}^{k}(m)=\sigma_{k} g_{j}^{k}(m)+\mu_{k} g_{j}^{k}(m-1)+\lambda_{k} g_{j}^{k}(m-2)
$$

Then it can be proved easily that the formal power series in $\varepsilon$.

$$
F_{j}^{k}(\varepsilon, m) \sim \varepsilon^{m-\sigma_{j}-\sigma_{k}} \sum_{s=0}^{\infty} H^{k}(s) g_{j}^{k}(m+s) \varepsilon^{s}
$$

satisfy the difference differential systems

$$
\begin{aligned}
\varepsilon \frac{d}{d \varepsilon}\left(\begin{array}{l}
F_{j}^{k}(\varepsilon, m) \\
F_{j}^{k}(\varepsilon, m-1)
\end{array}\right)= & \left(\begin{array}{cc}
0 & \mu_{k} \varepsilon \\
0 & 0
\end{array}\right)\left(\begin{array}{l}
F_{i}^{k}(\varepsilon, m) \\
F_{j}^{k}(\varepsilon, m-1)
\end{array}\right) \\
& +\left(\begin{array}{cc}
\lambda_{k} \varepsilon^{2} & 0 \\
\mu_{k} \varepsilon & \lambda_{k} \varepsilon^{2}
\end{array}\right)\left(\begin{array}{l}
F_{j}^{k}(\varepsilon, m-2) \\
F_{j}^{k}(\varepsilon, m-3)
\end{array}\right)
\end{aligned}
$$

and

$$
\begin{aligned}
\varepsilon \frac{d}{d \varepsilon}\left(\begin{array}{l}
F_{j}^{k}(\varepsilon, m) \\
F_{j}^{k}(\varepsilon, m-1)
\end{array}\right)= & \left(\begin{array}{cc}
\rho_{j}+m-A_{0} & A_{1}-\mu_{k} \\
0 & \rho_{j}+m-1-A^{0}
\end{array}\right)\left(\begin{array}{l}
F_{j}^{k}(\varepsilon, m) \\
F_{j}^{k}(\varepsilon, m-1)
\end{array}\right) \\
& +\left(\begin{array}{ll}
A_{2}-\lambda_{k} & 0 \\
A_{1}-\mu_{k} & A_{2}-\lambda_{k}
\end{array}\right)\left(\begin{array}{l}
F_{j}^{k}(\varepsilon, m-2) \\
F_{j}^{k}(\varepsilon, m-3)
\end{array}\right)
\end{aligned}
$$

Moreover $F_{j}^{k}(1, m)$ formally satisfy $(6.3)$

To prove that $F_{j}^{k}(1, m)$ have any analytical meaning, we have to eliminate $F_{j}^{k}(\varepsilon, m-2)$ and $F_{j}^{k}(\varepsilon, m-3)$ from the systems (6.12) and (6.13), and have to prove the convergence at $\varepsilon=1$ of the formal solutions (6.11) of the differential systems involving only $F_{j}^{k}(\varepsilon, m)$ and $F_{j}^{k}(\varepsilon, m-1)$, which will be written as

$$
\varepsilon \frac{d}{d \varepsilon}\left(\begin{array}{l}
F_{j}^{k}(\varepsilon, m) \\
F_{j}^{k}(\varepsilon, m-1)
\end{array}\right)=B(\varepsilon)\left(\begin{array}{l}
F_{j}^{k}(\varepsilon, m) \\
F(\varepsilon, m-1)
\end{array}\right)
$$

Subtracting (6.12) from (6.13), we have 


$$
\begin{array}{r}
\left(\begin{array}{cc}
\lambda_{k} \varepsilon^{2}+\left(\lambda_{k}-A_{2}\right) & 0 \\
\mu_{k} \varepsilon+\left(\mu_{k}-A_{1}\right) & \lambda_{k} \varepsilon^{2}+\left(\lambda_{k}-A_{2}\right)
\end{array}\right)\left(\begin{array}{l}
F_{j}^{k}(\varepsilon, m) \\
F_{j}^{k}(\varepsilon, m-1)
\end{array}\right) \\
=\left(\begin{array}{cc}
\rho_{j}+m-A_{0} & A_{1}-\mu_{k}-\mu_{k} \varepsilon \\
0 & \rho_{j}+m-1-A_{0}
\end{array}\right)\left(\begin{array}{l}
F_{j}^{k}(\varepsilon, m-2) \\
F_{j}^{k}(\varepsilon, m-3)
\end{array}\right)
\end{array}
$$

The determinant of the matrix on the left is clearly

$$
\begin{aligned}
D(\varepsilon)= & \left.\left\{\lambda_{k} \varepsilon^{2}+\left(\lambda_{k}-\lambda_{1}\right)\right\}^{2}\left\{\lambda_{k} \varepsilon^{2}+\lambda_{k}-\lambda_{2}\right)\right\}^{2} \cdots \\
& \left.\cdots\left\{\lambda_{k} \varepsilon^{2}+\lambda_{k}-\lambda_{n}\right)\right\}^{2}
\end{aligned}
$$

The only possible singularities of the system (6.14) are those values of $\varepsilon$ for which $D(\varepsilon)=0$, namely,

$$
\varepsilon=0, \pm \sqrt{\frac{\lambda_{j}-\lambda_{k}}{\lambda_{k}}} \quad(j \neq k)
$$

The character of the singular point $\varepsilon=0$ is determined by $M$. Iwano to be a regular singular point. Thus the formal solutions (6.11) are convergent in the closed unit disk where none but $\varepsilon=0$ is singular, and especially they are convergent at $\varepsilon=1$ thanks to our pentagonal condition.

This is not characteristic only for the singularities of rank one and two. Because the form of $D(\varepsilon)$ for the system

$$
t \frac{d X}{d t}=\left(A_{0}+A_{1} t+\cdots+A_{q} t^{q}\right) X
$$

is

$$
D(\varepsilon)=\left[\operatorname{det}\left\{\lambda_{k} \varepsilon^{q}-\left(\lambda_{k}-A_{q}\right)\right\}\right]^{q}
$$

for an eigenvalue $\lambda_{k}$ of the diagonal matrix $A_{q}$.

It is needless to remark that once the convergence of $F_{j}^{k}(1, m)$ is established, the corresponding connection problem for the difference system (6.3) is completely solved in convergent form. The remaining task is, then, to study the asymptotic behavior of the series of the form

$$
\sum_{m=0}^{\infty} \varphi(m) g_{j}^{k}(m) t^{m}
$$

for bounded $\varphi(m)$. 
The details of this section will be given in our subsequent study. A comparison of our method with the reduction of rank introduced by H.L. Turrittin is interesting in this direction.

\section{REFERENCES}

[1] K. O. Friedrichs, Special topics in Analysis. New York University 1953-54.

[3] K. Okubo, A global representation of a fundamental set of solutions and a Stokes phenomenon for a system of linear ordinary difierential equations, Jour. Math. Soc. Jap. 15 (1963), 268-288.

[3] H. L. Turrittin, Reduction of ordinary differential equations to the Birkhoff canonical form, Trans. Amer. Math. Soc., 107 (1963), 485-507.

[4] , Reducing the rank of ordinary differential equations, Duke Math. Jour. 30 (1963). 271-274.

[5] - Solvable related equations pertaining to turning point problems, Asymptotic solutions of differential equations and their applications, edited by A. Wilcox. (1964). 27-52.

[6] E. M. Wright, The asymptotic expansion of integral functions defined by Taylor series, Phil. Trans. Royal Soc. London, A238 (1940), 389-408. 